\title{
Calidad de vida en adultos jóvenes trasplantados renales con y sin depresión
}

\section{Quality of life in young adults with kidney transplants with and without depression}

\author{
Ma. Yazmín Aranda-Ramírez ${ }^{1}$, Rosa Ma. Rodríguez-Medina ${ }^{1,2,3 *}$, Brenda E. Franco-Corona ${ }^{1}$ \\ y Carlos F. Meza-García ${ }^{1,2,3}$ \\ ${ }^{1}$ Departamento de Enfermería y Obstetricia, Universidad de Guanajuato, Campus Guanajuato; ${ }^{2}$ Departamento de Enfermería y Obstetricia, Universidad de Guanajuato, \\ Campus León; ${ }^{3}$ Subdirección de Enfermería, Hospital Regional de Alta Especialidad del Bajío. León, Gto., México
}

\begin{abstract}
Background: Young adults with kidney transplants are a group susceptible to depressive symptoms, which can influence their quality of life. The objective was to evaluate the relationship between depression and quality of life in young kidney transplants from a tertiary care unit. Methods: Analytical and cross-sectional study, with convenience sampling. Forty hospitalized adults were included, quality of life was measured with the KDQOL-SF24 questionnaire (Kidney Disease Quality of Life-Short Form 24) and the Hamilton scale was applied to determine the level of depression. Results: The prevalence of depression was $70 \%$; participants with depression showed a lower quality of life in social function $(p=0.039)$ and mental health $(p=0.016)$. Conclusions: Young adults with kidney transplantation show a higher prevalence of depression compared to other age groups. It is suggested to identify patients with depression in a timely manner to increase their quality of life after kidney transplantation.
\end{abstract}

Key words: Kidney transplant. Quality of life. Depressive symptoms.

\section{RESUMEN}

Introducción: Los adultos jóvenes con trasplante renal son un grupo susceptible de presentar síntomas depresivos, lo cual puede influir en su calidad de vida. El objetivo fue evaluar la relación entre la depresión y la calidad de vida de jóvenes trasplantados renales de una unidad de tercer nivel. Métodos: Estudio analítico y de sección transversal, con muestreo por conveniencia. Se incluyeron 40 adultos hospitalizados, se midió la calidad de vida con el cuestionario KDQOL-SF24 (Kidney Disease Quality of Life-Short Form 24) y se aplicó la escala de Hamilton para conocer el nivel de depresión. Resultados: La prevalencia de depresión fue del 70\%; los participantes con depresión mostraron menor calidad de vida en la función social $(p=0.039)$ y salud mental $(p=0.016)$. Conclusiones: Los adultos jóvenes con trasplante renal muestran mayor prevalencia de depresión comparados con otros grupos etarios. Se sugiere identificar oportunamente a los pacientes que cursan con depresión para aumentar su calidad de vida posterior al trasplante renal.

Palabras clave: Trasplante renal. Calidad de vida. Síntomas depresivos.

\section{Correspondence:}

${ }^{*}$ Rosa Ma. Rodríguez-Medina

E-mail: anidem_2_2@hotmail.com
Date of reception: 09-09-2019

Date of acceptance: 14-09-2020

DOI: 10.24875/HMCM.19000229
Available online: $18-11-2020$

Hosp Med Clin Manag. 2020;13:103-8

2604-0018 / @ 2020 Mexican Regional Hospitals of High Specialty and Federal Hospitals. Published by Permanyer. This is an open access article under the CC BY-NC-ND license (http://creativecommons.org/licenses/by-nc-nd/4.0/). 


\section{INTRODUCCIÓN}

La enfermedad renal terminal (ERT) se ha convertido en un problema de salud pública que conlleva innumerables desafíos para los países en vías de desarrollo y que, además, conduce a un impacto importante en la calidad de vida ${ }^{1}$. De acuerdo con la Organización Mundial de la Salud (OMS), la calidad de vida es un concepto multidimensional que se refiere al bienestar físico, emocional y social, habitual o esperado de una persona².

El trasplante renal se considera como el tratamiento de elección para los pacientes con ERT debido a que ofrece una mejor calidad de vida comparado con otras terapias de reemplazo renal. Tamura, et al. observaron mayor calidad de vida en pacientes con trasplante renal comparados con pacientes en tratamiento de hemodiálisis ${ }^{3}$. En otro estudio, Costa, et al. midieron la calidad de vida de receptores renales después de seis meses de haber sido trasplantados y encontraron que esta fue similar a la calidad de vida de la población general ${ }^{4}$.

Por otra parte, estudios previos han identificado que la depresión es el trastorno psiquiátrico más frecuente en pacientes con ERT, aumentando en 2.7 veces el riesgo de mortalidad $^{5}$. Respecto a la prevalencia de depresión en receptores renales, Liu, et al. incluyeron una muestra de adultos mayores y encontraron una prevalencia del $23 \%^{6}$. En contraste, Lin, et al. observaron una prevalencia del $59.2 \%$ en pacientes con edad media de $47.4 \pm 12.3$ años ${ }^{7}$.

Los resultados anteriores son relevantes, dado que la depresión impacta negativamente en los resultados esperados del trasplante renal, debido a su asociación con la falta de adherencia al tratamiento farmacológico, estado nutricional deficiente, mayor número de ingresos hospitalarios y estancias más prolongadas ${ }^{8}$.

Cabe mencionar que la depresión es uno de los factores principales que afectan negativamente la calidad de vida del paciente con ERT ${ }^{9}$. Sin embargo, es escasa la información sobre la prevalencia de la depresión en receptores de trasplante renal ${ }^{10}$. Este conocimiento representaría sin duda el primer paso para establecer estrategias preventivas eficaces y específicas para los diferentes grupos de edad, ya que como resultado del incremento de la supervivencia de los niños con enfermedades crónicas, hay un notable aumento en la prevalencia de adultos jóvenes con estas patologías ${ }^{11}$.
Dado este contexto, el presente estudio se realizó con el objetivo de evaluar la relación entre la depresión y la calidad de vida de adultos jóvenes trasplantados renales en una unidad de tercer nivel. La información anterior permitirá proponer estrategias de intervención que ayuden a mejorar la supervivencia del paciente y del injerto renal.

\section{MÉTODOS}

\section{Diseño y población de estudio}

Estudio analítico y de sección transversal. Se realizó un muestreo por conveniencia no aleatorio que incluyó a 40 pacientes adultos con trasplante renal hospitalizados en el Servicio de trasplantes de una unidad de tercer nivel, durante el periodo de enero a abril de 2018. Los criterios de inclusión fueron: rango de edad entre 18 y 45 años, ambos sexos, con mínimo un mes de haber sido trasplantados, y aceptar participar en el estudio previo consentimiento informado. No se incluyó a los pacientes hemodinámicamente inestables y/o con alteración neurológica.

La recolección de datos se realizó por personal de enfermería capacitado, quien entrevistó a los pacientes hospitalizados en el Servicio de trasplantes durante el turno matutino. En primera instancia se solicitaron datos sociodemográficos para conocer la edad, sexo, estado civil y tiempo transcurrido después del trasplante renal.

\section{Nivel de depresión}

Se aplicó el cuestionario Hamilton Depression Rating Scale (HDRS-17), desarrollado por el psiquiatra Max Hamilton en 1950 y reconocido como el estándar de oro para medir la intensidad o gravedad de los síntomas depresivos. El cuestionario está integrado por 17 puntos con escala tipo Likert para evaluar la presencia de síntomas somáticos de depresión mediante la aplicación de una entrevista semiestructurada $^{12}$.

El cuestionario HDRS-17 se integra por 8 artículos con una escala de 5 puntos, mientras que los 9 artículos restantes se contestan con una escala de 3 puntos. Para obtener el resultado es necesario realizar la sumatoria total de las respuestas obtenidas, cuyo rango oscila entre 0 y $52^{13}$. Los puntos de corte recomendados 
Tabla 1. Dimensiones de calidad de vida del Cuestionario KDQoL-SF24 e interpretación de las puntuaciones obtenidas

\begin{tabular}{|c|c|c|c|}
\hline \multirow{2}{*}{$\begin{array}{l}\text { Dimensiones } \\
\text { KDQoL SF24 }\end{array}$} & & \multicolumn{2}{|c|}{ Interpretación de las puntuaciones } \\
\hline & & $\begin{array}{l}\text { Menor puntuación } \\
\text { (0 a } 100 \text { puntos) }\end{array}$ & $\begin{array}{l}\text { Mayor puntuación } \\
\text { (100 puntos) }\end{array}$ \\
\hline \multirow[t]{5}{*}{$\begin{array}{l}\text { Dimensiones de } \\
\text { salud física }\end{array}$} & Función física & $\begin{array}{l}\text { Limitación para llevar a cabo las actividades físicas } \\
\text { (caminar, subir escaleras, inclinarse, etc.) }\end{array}$ & Realización de actividades físicas sin problema \\
\hline & Rol físico & $\begin{array}{l}\text { Los problemas de salud interfieren con el trabajo u } \\
\text { otras ABVD }\end{array}$ & Realización de las ABVD sin problema \\
\hline & Dolor & $\begin{array}{l}\text { Referencia de dolor de intensidad variable y que } \\
\text { limita sus actividades }\end{array}$ & Sin dolor ni limitaciones debidas a este \\
\hline & Salud general & $\begin{array}{l}\text { Autovaloración negativa de la salud actual y en el } \\
\text { futuro }\end{array}$ & $\begin{array}{l}\text { Autovaloración positiva de la salud actual y en el } \\
\text { futuro }\end{array}$ \\
\hline & Vitalidad & Referencia de cansancio y agotamiento físico & Referencia de sentirse con vitalidad \\
\hline \multirow[t]{4}{*}{$\begin{array}{l}\text { Dimensiones de } \\
\text { salud mental }\end{array}$} & Función social & $\begin{array}{l}\text { Los problemas de salud interfieren en las } \\
\text { actividades sociales habituales }\end{array}$ & $\begin{array}{l}\text { Realización de actividades sociales habituales sin } \\
\text { problema }\end{array}$ \\
\hline & Rol emocional & $\begin{array}{l}\text { Los problemas emocionales interfieren con el } \\
\text { trabajo u otras ABVD }\end{array}$ & $\begin{array}{l}\text { Ningún problema con el trabajo u otras ABVD } \\
\text { debido a problemas emocionales }\end{array}$ \\
\hline & Salud mental & $\begin{array}{l}\text { Referencia de sentimiento de angustia } \\
\text { y/o depresión }\end{array}$ & $\begin{array}{l}\text { Sin referencia de sentimientos de angustia y/0 } \\
\text { depresión }\end{array}$ \\
\hline & Efectos del riñón & $\begin{array}{l}\text { Refiere que su salud es mucho peor ahora } \\
\text { que hace un año }\end{array}$ & $\begin{array}{l}\text { Refiere que su salud general es mucho mejor ahora } \\
\text { que hace un año }\end{array}$ \\
\hline
\end{tabular}

KDQOL-SF24: Kidney Disease Quality of Life-Short Form 24; ABVD: actividades básicas de la vida diaria.

Adaptada de Jansz, et al..$^{14}$.

para definir la gravedad de la depresión son los siguientes: sin depresión 0- 7, depresión leve 8-16, moderada 1723 y depresión severa $\geq 24$ puntos $^{12}$.

\section{Calidad de vida}

Se aplicó el cuestionario Kidney Disease Quality of LifeShort Form 24 (KDQoL-SF24), el cual ha sido ampliamente utilizado para evaluar la calidad de vida de personas con ERT. EI KDQoL-SF24 está integrado por 24 preguntas de opción múltiple que describen la calidad de vida en ocho dimensiones de salud física y mental, además de los efectos de la enfermedad renal ${ }^{14}$. Para obtener el resultado es necesario identificar los ítems que integran cada dimensión, y enseguida se recodifican las respuestas obtenidas de tal manera que una puntuación mayor siempre represente un estado de salud más favorable. Posteriormente se realiza una transformación en una escala de 0 a 100, donde una mayor puntuación representa una mejor calidad de vida (Tabla 1 ).

\section{Análisis estadístico}

Se integró una base de datos en el Statistical Package for the Social Sciences (SPSS) versión 21. Se evaluó la normalidad en la distribución de los datos mediante la prueba de bondad de ajuste de Kolmogorov-Smirnov con corrección de Lilliefors. Las variables cualitativas se reportaron con frecuencias y porcentajes mientras que las variables cuantitativas se presentaron con media y desviación estándar. Se compararon las puntuaciones de las dimensiones de calidad de vida en los participantes con y sin depresión mediante la prueba t para muestras independientes. Se aplicó el coeficiente de correlación de Pearson para evaluar la relación entre la depresión y las dimensiones de calidad de vida. Se aceptó un valor de $p<0.05$ para la significancia estadística.

\section{CONSIDERACIONES ÉTICAS}

El protocolo de investigación fue aprobado por el Comité de Investigación y el Comité de Ética en Investigación de la institución de salud con los números de registro CI/HRAEB/2017/040 y CEI-41-17, respectivamente. La información que proporcionaron los participantes se manejó de manera confidencial para preservar su identidad e integridad. Se cumplió con el Reglamento de la Ley General de Salud en materia de investigación para la salud; de acuerdo con el Art. 17 se consideró un estudio con riesgo mínimo ${ }^{15}$. Además, se apegó a los mandatos de la Declaración de Helsinki de $2013^{16}$. Cabe mencionar que se consideró la probabilidad de que alguna pregunta pudiera afectar la integridad emocional de los 
Tabla 2. Características de los adultos jóvenes trasplantados renales

\begin{tabular}{lcc}
\hline Características & $\mathbf{f}$ & $\mathbf{( \% )}$ \\
\hline Edad (años) & ${ }^{\star}$ & $27.9 \pm 9.7$ \\
Media (DE) & \multicolumn{2}{c}{} \\
Sexo & 23 & $(57.5)$ \\
Masculino & 17 & $(42.5)$ \\
Femenino & & \\
Estado civil & 28 & $(70.0)$ \\
Soltero & 9 & $(22.5)$ \\
Casado & 3 & $(7.5)$ \\
Unión libre & & \\
Tiempo después de trasplante renal & 27 & $(67.5)$ \\
$<3$ años & 13 & $(32.5)$ \\
$>3$ años & & \\
Nivel de depresión & 12 & $(30)$ \\
Sin depresión & 20 & $(50)$ \\
Depresión leve & 8 & $(20)$ \\
Depresión moderada & 0 & $(0)$ \\
Depresión severa & & \\
\hline
\end{tabular}

*Se presenta como media y desviación estándar.

participantes, por lo que se contó con el apoyo del Servicio de Psicología para que brindara terapia de contención en caso necesario.

\section{RESULTADOS}

\section{Características de los participantes}

En el presente estudio participaron 40 pacientes, con edad media de $27.9 \pm 9.7$ años, y se contó con mayor participación de hombres (57.5\%); la mayoría de los participantes refirió estar soltero (70\%) y tener menos de tres años de haber sido trasplantado (67.5\%). Además, la mayoría de los participantes (70\%) se encontró con depresión (Tabla 2).

Se exploró la distribución de los casos con y sin depresión conforme a su edad y tiempo postrasplante mediante una tabla de contingencia y no se observaron diferencias significativas. En la figura 1 se presenta la prevalencia de depresión de acuerdo con el sexo de los participantes, la cual fue mayor en hombres (57.1\%).

\section{Dimensiones de calidad de vida en los participantes con y sin depresión}

Se compararon las puntuaciones de las dimensiones de calidad de vida en los participantes con y sin depresión (Tabla 3). Los participantes con depresión mostraron
70

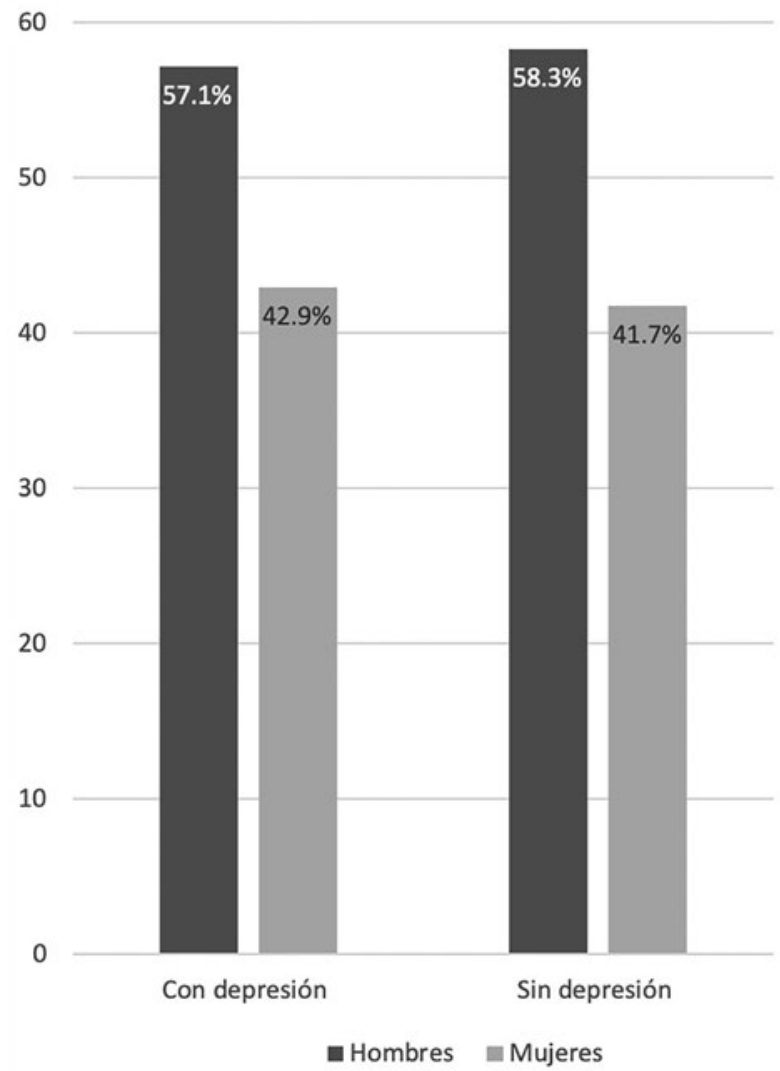

Figura 1. Prevalencia de depresión de acuerdo con el sexo de los participantes.

menor calidad de vida en las dimensiones de función social ( $p=0.039)$ y salud mental $(p=0.016)$.

Adicionalmente, se evaluó la correlación entre las dimensiones de calidad de vida y el nivel de depresión (Tabla 4). Se encontró relación entre el nivel de depresión y las tres dimensiones de salud mental: función social ( $p=0.004)$, rol emocional ( $p=0.017$ ) y salud mental ( $p=0.008)$.

\section{DISCUSIÓN}

El presente estudio permitió describir las características sociodemográficas de pacientes trasplantados en una unidad de tercer nivel de atención, la cual se encontró dentro de los primeros cinco establecimientos que realizaron más trasplantes de riñón en el 2019 de acuerdo con los registros del Centro Nacional de Trasplantes (CENATRA) ${ }^{17}$. 
Tabla 3. Dimensiones de la calidad de vida en los participantes con y sin depresión*

\begin{tabular}{lccc}
\hline Dimensiones KDQoL- SF24 & $\begin{array}{c}\text { Con depresión } \\
(\mathbf{n}=\mathbf{2 8})\end{array}$ & $\begin{array}{c}\text { Sin depresión } \\
(\mathbf{n}=\mathbf{1 2})\end{array}$ & Valor $\mathbf{p}$ \\
\hline Dimensiones de salud física & & & 0.250 \\
\hline Función física & $79.4 \pm 15.4$ & $82.3 \pm 14.4$ & 0.872 \\
Rol físico & $75.6 \pm 8.2$ & $75.9 \pm 8.5$ & 0.530 \\
Dolor & $82.9 \pm 5.6$ & $82.1 \pm 2.9$ & 0.607 \\
Salud general & $57.4 \pm 5.1$ & $59.5 \pm 5.9$ & 0.132 \\
Vitalidad & $82.9 \pm 3.5$ & $85.1 \pm 2.6$ & 0.039 \\
\hline Dimensiones de salud mental & & & 0.056 \\
\hline Función social & $70.1 \pm 4.5$ & $74.9 \pm 4.1$ & 0.016 \\
Rol emocional & $65.9 \pm 11.9$ & $69.9 \pm 9.4$ & 0.413 \\
Salud mental & $47.4 \pm 4.6$ & $53.1 \pm 4.4$ & $66.5 \pm 6.6$ \\
Efectos de la enfermedad renal & $64.3 \pm 8.6$ & \\
\hline
\end{tabular}

*Los valores se presentan como media \pm desviación estándar. Comparación de medias con la prueba t de Student para muestras independientes.

KDQOL-SF24: Kidney Disease Quality of Life-Short Form 24.

Tabla 4. Correlaciones entre el nivel de depresión y las dimensiones de calidad de vida*

\begin{tabular}{lcc}
\hline Dimensiones KDQoL- SF24 & $\begin{array}{c}\text { Coeficiente de } \\
\text { correlación }\end{array}$ & Valor p \\
\hline Dimensiones de salud física & & \\
\hline Función física & -0.296 & 0.064 \\
Rol físico & -0.032 & 0.847 \\
Dolor & 0.128 & 0.433 \\
Salud general & -0.138 & 0.394 \\
Vitalidad & 0.148 & 0.362 \\
\hline Dimensiones de salud mental & & \\
\hline Función social & -0.448 & 0.004 \\
Rol emocional & -0.376 & 0.017 \\
Salud mental & 0.414 & 0.008 \\
Efectos de la enfermedad renal & 0.215 & 0.071 \\
\hline
\end{tabular}

Se presentan los valores del coeficiente de correlación de Pearson.

KDQ0L-SF24: Kidney Disease Quality of Life-Short Form 24.

En este sentido, es un resultado preocupante que más de la mitad de los participantes haya presentado depresión, pero que podría explicarse dado el rango de edad de los receptores. De acuerdo con Malhi, et al., el inicio del primer episodio depresivo se presenta entre los 20 y 40 años. Además, en la segunda y tercera décadas de la vida se presenta un pico máximo en la prevalencia, con un incremento más discreto en la quinta y sexta décadas ${ }^{18}$.

Cabe mencionar que aunque el trasplante renal ofrezca mejores resultados comparado con otras terapias de sustitución, se pueden presentan situaciones como la incertidumbre sobre la salud o la insuficiencia de recursos económicos para costear el tratamiento inmunosupresor, que conllevan una tensión psicológica significativa incluso posterior al trasplante ${ }^{19}$. En los últimos años, la investigación relacionada con el trasplante renal no se encuentra centrada únicamente en la salud física de los pacientes, sino que existe un interés creciente en el estado psicológico de estos ${ }^{20}$.

Por otra parte, los resultados respecto al efecto de la depresión en la calidad de vida de personas con ERT coinciden con los hallazgos reportados en la literatura. Teles, et al. incluyeron a pacientes con edad media de $46.6 \pm 15.4$ años y en tratamiento de hemodiálisis, observando una prevalencia de depresión del 29\% y menores puntuaciones en todos los dominios SF-36 en los pacientes con depresión. Además, identificaron factores asociados con el deterioro de la calidad de vida como el número de comorbilidades, la presencia de diabetes, anemia e hipoalbuminemia ${ }^{21}$.

Finalmente, se destacan como fortalezas del presente estudio el aporte al conocimiento sobre la prevalencia de depresión y su impacto en la calidad de vida de pacientes adultos jóvenes trasplantados renales, dado que las investigaciones en torno a esta temática son escasas. Además, se destaca la importancia de diagnosticar oportunamente al paciente trasplantado que cursa con depresión para ofrecer apoyo psicológico durante el proceso de adaptación y fragilidad psicológica posterior al trasplante $22-24$, así como intervenciones educativas para orientarlo en su autocuidado 25 . 
Dentro de las limitaciones observadas se identifica el diseño transversal del estudio, el cual no permitió establecer una relación causal entre las variables de estudio. Es importante también mencionar que la información proporcionada, por las características sociodemográficas, no permitió identificar factores predictores de la depresión y la calidad de vida de los participantes ya que, como se reporta en los resultados, no se observaron diferencias en su distribución.

\section{CONCLUSIONES}

En el presente estudio los adultos jóvenes trasplantados renales mostraron mayor prevalencia de depresión comparados con otros grupos etarios. Respecto a la evaluación de la calidad de vida, los participantes con depresión obtuvieron menor puntuación en las dimensiones de función social y salud mental. Por ello es posible concluir que los adultos jóvenes que cursan con ERT y que reciben un trasplante renal son susceptibles de presentar problemas emocionales como la depresión, la cual condiciona un deterioro de su calidad de vida.

\section{FINANCIAMIENTO}

Los autores no recibieron patrocinio para llevar a cabo este artículo.

\section{CONFLICTO DE INTERESES}

Los autores declaran no tener conflicto de intereses alguno.

\section{RESPONSABILIDADES ÉTICAS}

Protección de personas y animales. Los autores declaran que para esta investigación no se han realizado experimentos en seres humanos ni en animales.

Confidencialidad de los datos. Los autores declaran que en este artículo no aparecen datos de pacientes.

\section{Derecho a la privacidad y consentimiento informado.} Los autores declaran que en este artículo no aparecen datos de pacientes.

\section{BIBLIOGRAFÍA}

1. Zazzeroni L, Pasquinelli G, Nanni E, Cremonini V, Rubbi I. Comparison of quality of life in patients undergoing hemodialysis and peritoneal dialysis: a systematic review and meta- analysis. Kidney Blood Press Res. 2017;42(4):717-27.

2. Cardona- Arias JA, Higuita- Gutiérrez LF. Aplicaciones de un instrumento diseñado por la OMS para la evaluación de la calidad de vida. Rev Cuba Salud Pública. 2014;40(2):175-89.

3. Tamura Y, Urawa A, Watanabe S, Hasegawa T, Ogura T, Nishikawa K, et al. Mood status and quality of life in kidney recipients after transplantation. Transplant Proc. 2018;50(8):2521-5.

4. Costa- Requena G, Cantarell MC, Moreso F, Parramon G, Seron D. Health related quality of life in renal transplantation: 2 years of longitudinal followup. Med Clínica (English Ed). 2017;149 (3):114-8.

5. Barros A, Costa BE, Mottin CC, Avila DO. Depression, quality of life, and body composition in patients with end-stage renal disease: a cohort study. 2016:(10):301-6.

6. Wang WL, Liang S, Zhu FL, Liu JQ, Wang SY, Chen XM, et al. The prevalence of depression and the association between depression and kidney function and health-related quality of life in elderly patients with chronic kidney disease : a multicenter cross-sectional study. Clin nterv Aging. 2019;14:905-13.

7. Lin X, Lin J, Liu H, Teng S, Zhang W. Depressive symptoms and associated factors among renal-transplant recipients in China. Int J Nurs Sci. 2016;3(4):347-53

8. Murillo- Zamora E, Macías- de la Torre AA, Higareda- Almaraz MA. Prevalencia de depresión entre pacientes con enfermedad terminal en hemodiálisis de mantenimiento. Rev Med Inst Mex Seguro Soc. 2016;54(4):429-33.

9. Perales Montilla CM, Duschek S, Reyes del Paso GA. Calidad de vida relacionada con la salud en la enfermedad renal crónica: relevancia predictiva del estado de ánimo y la sintomatología somática. Nefrologia. 2016;36(3):275-82

10. Palmer S, Vecchio M, Craig JC, Tonelli M, Johnson DW, Nicolucci A, et al. Prevalence of depression in chronic kidney disease : systematic review and meta-analysis of observational studies. Kidney Int. 2013;84(1):179-91.

11. Agredo MA, Santamaría MP, Restrepo JM. Transición en adolescentes con trasplante renal: El paso de atención pediátrica al cuidado de adultos. Rev Colomb Nefrol. 2016:3(2):120-3.

12. Sharp R. The Hamilton rating scale for depression. Occup Med 2015;65(4):340

13. Bobo W, Angleró GC, Jenkins G, Hall- Flavin DK, Weinshilboum R, Biernacka JM. Validation of the 17-item Hamilton Depression Rating Scale definition of response for adults with major depressive disorder using equipercentile linking to Clinical Global Impression scale ratings: analysis of Pharmacogenomic Research Network Antidepressant Medication Pharmacogenomic Study (PGRN- AMPS) data. Hum Psychopharmacol. 2016;31(3):185-92.

14. Jansz TT, Bonenkamp AA, Boereboom FTJ, van Reekum FE, Verhaar MC, van Jaarsveld BC. Health-related quality of life compared between kidney transplantation and nocturnal hemodialysis. PLoS One. 2018;13(9):1-11.

15. Secretaría de Salud. Reglamento de la Ley General de Salud en materia de investigación para la salud. Publicada en el DOF el 2 de abril de 2014.

16. Declaración de Helsinki de la Asociación Médica Mundial. Principios éticos para las investigaciones médicas en seres humanos [Internet]. Asociación Médica Mundial; 2014 [consultado en agosto de 2019]. Disponible en: http://www.wma.net/es/30publications/10policies/b3/

17. CENATRA. Reporte anual 2019 de donación y trasplantes en México [Internet]. México: Secretaría de salud; 2019. Disponible en: https://www.gob. $\mathrm{mx} / \mathrm{cms} / \mathrm{uploads} /$ attachment/file/528301/Presentacion_anual_2019.pdf

18. Malhi GS, Mann JJ. Depression. Lancet. 2018;392:2299-312.

19. Moreno F, Mora SL, Castelblanco C, Molina C, Ortíz LA. Trastorno emocional en el paciente renal trasplantado. Enferm Nefrol. 2016;19(2):147-53.

20. Mena-Nájera A, Barragán-Fuentes AG, Gómez de Regil L. Intervenciones psicológicas en pacientes con trasplante renal. Evid Med Invest Salud. 2014;7(2):70-5.

21. Teles F, Amorim de Albuquerque AL, Freitas Guedes Lins IK, Carvalho Medrado P, Falcão Pedrosa Costa A. Quality of life and depression in haemodialysis patients. Psychol Heal Med. 2018;23(9):1069-78.

22. Pistorio ML, Veroux M, Trigona $C$, Patanè $M$, Lo Bianco $S$, Cirincione $G$, et al. Psychological and emotional aspects in living donor kidney transplantation. Transplant Proc. 2019;51(1):124-7

23. Suzuki R, Nakamiya $Y$, Watanabe M, Ando E, Tanichi M, Koga M, et al. Relationship between stress coping mechanisms and depression in kidney transplant recipients. Transplant Proc. 2019;51(3):761-77.

24. Czyżewski, Frelik P, Wyzgał J, Szarpak. Evaluation of quality of life and severity of depression, anxiety, and stress in patients after kidney transplantation. Transplant Proc. 2018;50(6):1733-7.

25. Urstad KH, Wahl AK, Andersen MH, Øyen O, Hagen KB. Limited evidence for the effectiveness of educational interventions for renal transplant recipients. Results from a systematic review of controlled clinical trials. Patient Educ Couns. 2013;90(2):147-54 